\title{
L'échelle du paradoxe ou la contextualisation au centre du jugement de figuralité
}

Paradox and Contextualization: An Essential Link to be Considered as a Figure of Speech

\section{Agathe Mezzadri}

\section{OpenEdition}

Journals

\section{Édition électronique}

URL : http://journals.openedition.org/pratiques/2564

DOI : 10.4000/pratiques.2564

ISSN : 2425-2042

Éditeur

Centre de recherche sur les médiations (CREM)

Référence électronique

Agathe Mezzadri, «L'échelle du paradoxe ou la contextualisation au centre du jugement de figuralité », Pratiques [En ligne], 165-166 | 2015, mis en ligne le 01 octobre 2015, consulté le 01 mai 2019. URL : http://journals.openedition.org/pratiques/2564 ; DOI : 10.4000/pratiques.2564

Ce document a été généré automatiquement le 1 mai 2019.

(c) Tous droits réservés 


\title{
L'échelle du paradoxe ou la contextualisation au centre du jugement de figuralité
}

\author{
Paradox and Contextualization: An Essential Link to be Considered as a Figure of
} Speech

Agathe Mezzadri

1 Le paradoxe peut-il être une figure ? C'est la question que soulève J.-C. Margolin (1988 : 14) pour y répondre par la négative : « Le paradoxe est au service de la rhétorique et de la dialectique, ou se situe à leur intersection : il ne saurait être considéré comme une figure (au sens classique) de la seule rhétorique ». C'est ce "sens classique » que nous nous proposons d'interroger dans la perspective des travaux pragmatiques de $\mathrm{M}$. Bonhomme (1998: 7) où la figure du discours est une « forme discursive marquée, libre et mesurable qui renforce le rendement des énoncés ». Cette définition nous intéresse particulièrement dans la mesure où elle associe technicité, expressivité stylistique et contextualisation dans le jugement de figuralité.

Quels sont, au préalable, les arguments pour écarter le paradoxe de l'univers figural «classique » et en faire un concept trouvant sa réalisation formelle dans d'autres figures ? J.-C. Margolin (1988:7) s'appuie sur sa supposée absence des rhétoriques depuis l'Antiquité, son manque de "critères formels et objectifs", le caractère fluctuant de la notion de doxa ainsi qu'une proximité problématique avec l'oxymore.

3 Mais en conservant cette perspective "classique ", on s'aperçoit que la démonstration prend appui sur un examen partiel des rhétoriques antiques, de la notion de doxa et du modèle linguistique du paradoxe. Tout d'abord, une recherche approfondie révèle l'existence d'au moins deux figures antiques du paradoxe. La première, macrostructurale, se trouve chez les traducteurs d'Hermagoras et Aristote dans les cinq «figures de la controverse » du genre judiciaire ${ }^{1}$. Il suffit de considérer, à l'instar du Pseudo-Augustin ou de Fortunatianus d'une part, de F. Douay-Soublin, d'autre part, que ces skhếmata correspondent bien à des figurae $e^{2}$ latines et à nos « figures »3. La parádoxon skhếma choque 
la conception de la justice de l'audience. La seconde, microstructurale, correspond, elle, à une surprise due à l'emploi d'un mot parce qu'il est familier, forgé sur un autre mot ou employé de façon inadaptée par rapport au contexte. Démétrios (1993 : 43-44) et Cicéron (1971, LIV, 207 : 85-86) reprennent cette définition remontant au moins à Aristote (1973 : Livre III, 1412 a, 26 à 32, 69) sans lui attribuer le terme " paradoxe ». Mais surtout, deux auteurs latins tardifs - Julius Rufinianus et Isidore de Séville, sans doute jamais évoqués parce que non traduits - l'attachent, eux, au mot « paradoxe » et aux « figures de mots » ${ }^{4}$. Le deuxième argument de non-figuralité du paradoxe repose sur la bisémie de la doxa. Toutefois, que la doxa soit rationnelle (logique commune) ou sociale (opinion commune), elle peut toujours se définir comme une vérité apparente : apparente parce qu'évidente dans un cas, parce que circulant sans preuve dans l'autre. Elle n'empêche donc pas la conceptualisation du paradoxe $e^{5}$ que nous définissons univoquement sur le plan référentiel comme la "contradiction d'une vérité apparente " ${ }^{6}$, ouvrant la voie à une modélisation linguistique unique sur le plan formel. La formalisation du paradoxe constitue, en effet, le troisième problème soulevé par l'approche classique. À cet égard, une matrice a été proposée par M. Carel et O. Ducrot (1999b) :

Sont " paradoxaux » un enchainement d'énoncés, un énoncé ou un mot, qui réalisent, de façon interne, un aspect argumentatif A DONC (resp. POURTANT) B, alors que la signification " intrinsèque " de $A$ ne comporte pas cet aspect et, en outre, comporte l'aspect A POURTANT (resp. DONC) B (ibid. : 27) :

«Nous retrouvons bien, dans ce schème, le concept de vérité apparente dans la "signification "intrinsèque" qui peut correspondre à la doxa des juges ou à la suite attendue de la phrase des définitions antiques. Les principaux problèmes posés par l'approche taxinomique semblent alors se résoudre aisément.

Néanmoins, d'autres questions attachées au jugement de figuralité du paradoxe demeurent : sa taille, ses manifestations syntaxico-lexicales ainsi que ses rapports avec d'autres figures comme l'oxymore ou le paradoxisme. Sur ces points, seule l'approche des figures du discours permet une réflexion cohérente. Bien plus, la notion de contextualisation qui lui est attachée ${ }^{7}$ nous permet de considérer le paradoxe, non pas comme une figure problématique mais comme un parangon de figure du discours. Cette contextualisation s'avère, en effet, essentielle dans deux domaines : l'interaction avec les autres figures, d'une part, la précision du schème linguistique du paradoxe, d'autre part ».

\section{L'échelle du paradoxe, un contexte d'hybridation figurale}

5 Le paradoxe se distingue par des empans extrêmement divers qui ont amené les manuels à ne pas l'envisager comme une figure, ou uniquement de façon microstructurale, comme une figure de mots nommée parfois " paradoxisme » ou « antilogie »(Ricalens Pourchot, 2003; Gorp, 2001: 349; Bergez, Geraud \& Robrieux, 1994: 284), ou, à l'inverse, uniquement de façon macrostructurale, comme une figure de pensée ${ }^{8}$. En effet, accepter les différentes tailles du paradoxe, c'est remettre en question la fameuse opposition héritée de la Rhétorique à Herennius ${ }^{9}$ et fondatrice de nombreuses approches taxinomiques. Pourtant, c'est bien ignorer l'une ou l'autre de ses facettes que de le faire. Pour O. Ducrot et M. Carel (1999b), l'expression paradoxale peut porter sur « un mot, un syntagme, ou un discours " (ibid. : 6) et même un « un énoncé ou un enchaînement d'énoncés " (ibid. : 27). M. Tutescu (1996: 79-82), quant à elle, propose d'étudier son " échelle». Mais si elle ne dépasse pas le stade de la séquence paradoxale (qu'elle nomme paradoxe filé), nous 
proposons de l'étendre au texte et même au hors-texte, dans le cadre propice des figures du discours.

6 À l'origine, le mot paradoxal suit les exemples de "masochiste » et de "casse-cou » (Carel, Ducrot, 1999a). En montant dans l'échelle, le syntagme paradoxal se confond parfois avec l'oxymore comme le «torrent immobile» de P. Claudel (1965: 263) ${ }^{10}$. Puis vient la proposition paradoxale, celle qui adopte explicitement les dimensions de la formulation en DONC ou POURTANT : «J'aime assez mon amant pour renoncer à lui »", autrement dit «J'aime DONC je quitte » au lieu de "J'aime POURTANT je quitte ». Au-delà de la proposition paradoxale, l'enchainement de plusieurs paradoxes peut nous amener à la séquence paradoxale. L'échelle s'étend même au texte paradoxal, dans la célèbre Lettre sur la comète de P. Bayle qui développe le paradoxe de l'athée vertueux (2007) ou encore l'éloge paradoxal que M. Bonhomme (1998: 82) évoque comme figure. Le schème atteint, enfin, à la théorie paradoxale, en dehors des limites du texte, la doctrine du pur amour de Fénelon, par exemple, qui parcourt son œuvre. Le problème face à la doxa (culturelle) de l'époque incarnée par Bossuet peut se formuler ainsi: «œuvres DONC salut » à quoi Fénelon répond « œuvres POURTANT non salut ».

7 Précisons que nous ne parlons pas de registre paradoxal pour ces empans maximaux dans la perspective de M. Bonhomme. En effet, il semble difficile d'identifier un "macro-acte illocutoire $»^{12}$ systématique. S'il s'agit souvent de choquer ou déstabiliser l'auditoire, ce n'est évidemment pas la visée des paradoxes mystiques dont la fonction est davantage cognitive ou émotive et moins centrée sur le destinataire.

8 Enfin, cette conception semble conforme à la perspective aristotélicienne puisque les cinq occurrences du terme dans $\mathrm{La}$ Rhétorique dessinent un parcours allant du «mot inattendu " que l'on a évoqué, à la conclusion paradoxale d'un enthymème ${ }^{13}$, à la sentence paradoxale ${ }^{14}$ (déjà dans la Rhétorique à Alexandre, Pseudo-Aristote, 2002, $1430 \mathrm{~b}$ : 47-48), au discours tout entier de l'adversaire que, par conséquent, l'on interroge ${ }^{15}$ et enfin à l'affaire paradoxale précédemment analysée.

9 Ainsi, l'échelle du paradoxe se laisse appréhender comme une figure du discours unique par l'identification d'un même schème bien "mesurable $»^{16}$ et en cohérence avec l'héritage antique. Mais ce qui nous semble éminemment intéressant, ce sont les « variations libres » qui font de cette matrice linguistique un contexte figural inédit. Loin d'être uniquement "permises ", ces variations semblent inévitables comme le suggère d'emblée le cas de la séquence paradoxale :

«Quand je dis de l'être infini qu'il est l'être simplement, sans rien ajouter, j'ai tout dit. Sa différence, c'est de n'en avoir point. Le mot d'infini, que j'ai ajouté, ne lui donne rien d'effectif. C'est un terme presque superflu, que je donne à la coutume $»^{17}$

Le paradoxe ici consiste dans le fait de dire « je définis une entité (Dieu) DONC je ne lui attribue aucune qualité » s'opposant à une doxa qui serait «je définis une entité POURTANT je ne lui attribue aucune qualité». On remarque alors de nombreuses manifestations de ce paradoxe séquentiel : par exemple, «sans rien ajouter, j'ai tout dit ", où se mêlent parallélisme antonymique, antithèse et proposition paradoxale (« rien dire DONC tout dire " contre la doxa "rien dire DONC dire rien»). Ailleurs, l'antanaclase rejoint la proposition paradoxale ( "Quand je dis de l'être infini qu'il est l'être simplement»). On peut évoquer encore une autre proposition paradoxale ( $\mathrm{Sa}$ différence, c'est de n'en avoir point ») ou encore la référence à la doxa dans la «coutume ». Ainsi, le paradoxe se mêle à d'autres procédés, les engendre mais aussi repose sur eux. 
11 Mais loin de l'éloigner du domaine figural, cette ambigüité l'y inscrit pleinement. M. Bonhomme (2002: 9) consacre, en effet, un numéro de la revue Semen à cette caractéristique " prépondérante » voire "fondamentale » même si non systématique des figures du discours. L'une des dimensions de cette ambigüité est métalinguistique. Elle concerne l'« épineux problème de la relativité et de la gradualité des figures, qu'il s'agisse de leur statut ou de leur catégorisation ", autrement dit : le jugement de figuralité. En effet, une saillance donnée peut être perçue ou non comme une figure ${ }^{18}$ mais aussi comme telle ou telle figure tout à la fois (ibid.: 13) car "une même figure peut être simultanément antithétique, paradoxale, métaleptique...» (ibid.: 2002: 57). Ainsi, la figure est en elle-même un contexte d'hybridation figurale.

Ce qui est éminemment intéressant avec le paradoxe, c'est le caractère prédiscursif, pour ne pas dire essentiel, de cette hybridité. Notre procédé pose, en effet, ce problème dès sa conceptualisation théorique quand d'autres ne le soulèveront qu'en discours. On peut convoquer, à cet égard, la forte proximité du paradoxe avec certaines figures, notamment le paradoxisme et l'oxymore. Mais oxymore et paradoxe semblent indémêlables sur le plan théorique ${ }^{19}$ quand le terme paradoxisme se révèle peu opportun ${ }^{20}$. Nous pensons plutôt que c'est la diversité de ses empans qui fait de l'échelle du paradoxe un contexte propice à l'hybridité figurale, notre séquence paradoxale précédente se réalisant dans différentes figures dont celle, justement, de la proposition paradoxale.

Il convient de préciser, à cet égard, que l'interaction se produit dès les étapes minimales de l'échelle du paradoxe : le syntagme et la proposition. Que ce soit sous sa forme verbale - «se défier de soi-même »- ou nominale - «la défiance de soi-même », le syntagme récurrent des Aventures de Télémaque ${ }^{21}$, des ouvrages spirituel ${ }^{22}$ et de la correspondance ${ }^{23}$ de Fénelon se laisse envisager comme un lieu d'interaction figurale. En effet, la complémentation réalise une métaphore (faisant passer le sémantisme du verbe du domaine des relations humaines à celui de la lutte intérieure), une antithèse (entre extériorité et intériorité) et un énoncé culturellement paradoxal (la suite doxale attendue: «soi-même DONC confiance » ou encore "soi-même POURTANT défiance » étant remplacée par "soi-même DONC défiance »). De la même manière, la proposition est aussi un lieu d'oscillation entre, par exemple, paradoxe et antanaclase ( on ne veut plus les vertus comme vertus $»^{24}$ ), paradoxe, polyptote et parallélisme ( Je ne puis connaître l'infini que par une connaissance finie $»^{25}$ ) ou encore paradoxe, antimétabole, réduplication et chiasme ( Ne peut-on pas craindre de s'être trompé, sans s’être trompé en effet? $»^{26}$ ).

14 Ainsi, si la perspective des figures du discours accueille favorablement l'échelle du paradoxe, c'est en y accentuant une dimension prépondérante. Par son originalité structurale, la figure devient dès son approche théorique un contexte d'hybridation figurale majeure. Dans une tout autre perspective, le contexte du paradoxe est à envisager comme une composante essentielle de sa figuralité: sans amputation d'un élément de contexte, le paradoxe n'existe pas.

\section{Une nécessaire amputation du contexte discursif : paradoxe, pseudoparadoxe et style paradoxal}

15 Voltaire (1957: 1001) rappelle la nécessité du contexte pour les limites maximales de l'échelle du paradoxe, par exemple le «livre» : «Il fallut que Bayle écrivit contre le 
préjugé vulgaire un livre fameux, que les progrès de la raison ont rendu aujourd'hui moins piquant qu'il ne l'était alors $»^{27}$. Depuis, toutes les approches descriptives ont insisté sur le rôle du contexte dans le caractère éphémère et conjoncturel de ces paradoxes aux larges empans. Toutefois, il nous semble important de rappeler que cette caractéristique ne se limite pas à la macrostructure des textes, œuvres ou théories paradoxaux, bien au contraire.

Déjà, chez Aristote, le lien consubstantiel entre paradoxe microstructural et contextualisation apparait. Dans le passage de La Rhétorique consacré aux enthymèmes, le rhéteur suggère que c'est une abstraction du contexte qui les rend paradoxaux :

«Par exemple, une femme ayant renversé sous elle son propre fils à force de l'embrasser, on crut qu'elle faisait l'amour avec le jeune homme; la cause expliquée, la suspicion disparut $»^{28}$.

Sachant que l'enthymème est un syllogisme tronqué parce que la majeure, doxale, est connue de tous, le paradoxe mis en valeur ici apparait comme son symétrique inverse : une proposition, inconnue de tous, est à rétablir. C'est pourquoi, nous proposons d'identifier dans l'amputation d'un élément de contexte une caractéristique nécessaire du véritable paradoxe linguistique. Nous utiliserons la notion de " microcontexte implicite " de M. Riffaterre (1996: 151) :

«Le paradoxe [...] frappe sans avertir. Il est donc impossible de l'opposer à un microcontexte verbal explicite. Comme la transgression est néanmoins perçue, j'en conclus que le lecteur la perçoit en fonction d'un microcontexte implicite: la transgression se manifeste par rapport à ce que les mots ne disent pas mais qu'ils infèrent nécessairement, c'est-à-dire par rapport à leurs présuppositions ».

Toutefois, ces «présuppositions" correspondent à la suite doxale attendue et contrecarrée que nous avons analysée dans le modèle de $\mathrm{M}$. Carel et $\mathrm{O}$. Ducrot, pas à l'élément de contexte à rétablir selon Aristote. Nous ajoutons donc que la résolution du paradoxe, ce qui l'éloigne de la simple contradiction comme dans les exemples aristotéliciens, repose sur un deuxième "microcontexte implicite ». Sans ce caractère suggestif, la résolution du paradoxe est donnée par le texte, autorisant donc des lectures non paradoxales. Une citation tirée de La Démonstration de l'existence de Dieu permet de l'expliquer :

"Que les hommes accoutumés à méditer les vérités abstraites, et à remonter aux premiers principes, connaissent la Divinité par son idée ; c'est un chemin sûr pour arriver à la source de toute vérité. Mais plus ce chemin est droit et court, plus il est rude, et inaccessible au commun des hommes [qui dépendent de leur imagination]. C'est une démonstration si simple, qu'elle échappe par sa simplicité aux esprits incapables des opérations purement intellectuelles. Plus cette voie de trouver le premier Etre est parfaite, moins il y a d'esprits capables de la suivre $"^{29}$.

Nous pourrions aisément conserver le passage en italique pour le faire figurer au chapitre «paradoxe»d'un manuel ou dictionnaire. Nous illustrerions alors sa dimension polyphonique (Tutescu, 1996) dans laquelle la matrice de M. Carel et O. Ducrot est appliquée à une pluralité d'énonciateurs. En effet, l'énonciateur (E1) qui correspond à la norme, qu'elle soit issue de croyances ou du sémantisme des mots, ouvre un monde potentiel (M1), extension du monde tel qu'il est (M0). Dans (M1), à la proposition « plus ce chemin est droit et court » répond la consécution doxale " plus il est aisé au commun des hommes ", «microcontexte implicite» ou "présupposition» de Riffaterre. Mais cette attente est contrecarrée par un énonciateur (E2) qui soutient la thèse contraire dans un univers de croyance contrefactuel (M2). (E2) donne alors pour VRAIE une proposition qui, 
dans (M0), est admise pour FAUSSE : " plus il est rude, et inaccessible au commun des hommes ». univers de croyance (M3) et responsable de la signification implicite. Ce tiers résout le paradoxe en rétablissant un deuxième élément de "microcontexte implicite ", à savoir que la ligne droite est difficile uniquement pour le « commun des hommes », c'est-à-dire les non-philosophes dont la pensée spirituelle emprunte les détours de l'imagination. Ainsi, le paradoxe est bien un énoncé polyphonique mais qui engage nécessairement le lecteur dans deux et non pas un seul moment de coénonciation reposant sur les implicites $\mathrm{du}$ texte: l'identification d'une suite doxale implicite et contrecarrée, d'abord, la résolution de cette contradiction par le rétablissement d'un élément de contexte aussi implicite, ensuite.

C'est dans cette perspective que le paradoxe est d'autant plus rigoureux linguistiquement que le deuxième microcontexte (celui qui permet de lever la contradiction de la doxa) demeure effectivement implicite, totalement abstrait, comme chez Aristote. Dans notre exemple, le troisième énonciateur (E3) apparait entre les crochets : donné par le texte, il est simplement remarqué mais pas rétabli par le lecteur. Si mettre au jour un mécanisme de mouvement paradoxal relève bien de la compétence du lecteur, seul le premier microcontexte (la doxa contrecarrée) demeure un véritable implicite du texte. Du point de vue théorique, nous parlerons plus volontiers de pseudoparadoxe (Carel, Ducrot, 1999a : 25) pour ces formes engageant moins le lecteur dans la coénonciation que les véritables mécanismes à double microcontexte implicite comme l'exemple suivant :

«Un homme peut raisonner avec un autre homme, mais avec Dieu il n'y a qu'à prier, qu'à s'humilier, qu'à écouter, qu'à se taire, qu'à suivre aveuglément. Ce sacrifice de notre raison est le seul usage que nous puissions faire de notre raison même, qui est faible et bornée. Il faut que tout cède quand la raison suprême décide ${ }^{30}$.

Dans cette citation, le paradoxe repose sur l'équivalence entre "seul usage " et « sacrifice » ou énoncé en DONC : « exploiter un élément (la raison) DONC le détruire ». Accentué par la négation exceptive, la répétition du mot « raison » et l'adjectif de l'ipséité « même », il indique une contradiction totale : la seule façon de bien réfléchir est de ne le faire pas. Et si les allusions cotextuelles mettent sur la voie d'une résolution de cette erreur apparente, elles ne la fournissent pas explicitement. Il revient alors au lecteur de donner un caractère déterminatif à la subordonnée relative " qui est faible et bornée » et de la confronter à la raison divine qualifiée hyperboliquement de "suprême ", sur le modèle de la première phrase qui oppose les relations entre différentes raisons humaines, d'une part, et entre raison humaine et raison divine, d'autre part. En conférant tous ses présupposés à la sentence finale ( Il faut que tout cède quand la raison suprême décide »), il peut alors postuler un transfert de l'homme vers Dieu, justifiant l'amuïssement de ses facultés de pensée comme seule action possible. En effet, loin d'empêcher la réflexion de l'homme, le sacrifice de sa raison lui ouvre, au contraire, la voie à la plus extraordinaire des facultés de raisonnement: celle de Dieu. Ce microcontexte étant bien implicite, il sollicite largement l'acte de coénonciation, raison pour laquelle l'énoncé est strictement paradoxal. Mais l'impression de paradoxe est-elle toujours corrélée à sa rigueur linguistique?

Sur ce point, la question de la compétence du lecteur intervient grandement. Surtout, il semble possible d'identifier une dissimilation des axes de la linguistique et de la stylistique. En effet, certains pseudoparadoxes stylistiquement très forts, notamment 
ceux s'hybridant avec des figures d'opposition binaire, sont davantage activés comme paradoxaux que d'autres linguistiquement plus précis comme "Cette valise est légère, Jean ne pourra pas la porter " (Bruxelles, Ducrot \& Raccah, 1995 : 107-108). Et même, le paradoxe linguistique peut s'affaiblir à mesure que le style paradoxal augmente. Un pseudoparadoxe tiré des Dialogues des morts de Fénelon le suggère :

"Quand on sait goûter ces plaisirs simples de la pure nature, ils ne s'usent jamais, et on n'en manque point ; mais quand on les méprise, on a beau être riche et puissant, on manque de tout, [car on ne peut jouir de rien] $\aleph^{31}$.

Dans cet exemple, le troisième énonciateur (E3) constitue une voix du texte fournissant le deuxième "microcontexte » entre crochets : le fait de "ne jouir de rien " explique en quoi l'on peut être «riche et puissant» et «manquer de tout». Pourtant, la valeur stylistique du paradoxe semble justement renforcée par la présence de cette proposition. En effet, le parallélisme « manquer de tout / jouir de rien » et l'antithèse entre " tout » et « rien » accentuent l'effet de paradoxe en même temps qu'ils constituent l'élément de sa résolution. On voit donc ici se dessiner une nuance nette entre les axes de la linguistique et de l'esthétique où, proportionnellement, la force stylistique du paradoxe, sa qualité de figure de style, augmente à mesure que le paradoxe s'éloigne de sa modélisation linguistique, de son marquage formel comme figure $d u$ discours. C'est dans cet ordre d'idées que M. Bonhomme (voir note 16) évoque la distinction des deux plans et, partant, celle des figures $d u$ discours et des figures de style:

«Les "figures du discours" deviennent des "figures de style" lorsque diverses spécifications s'exercent sur leurs paramètres linguistiques de base. D'un côté, il faut que leurs variations libres manifestent une réelle créativité verbale, source d'opacification du sens, vis-à-vis de l'arrière-plan standardisé de la langue. D'un autre côté, il convient que leurs schèmes saillants s'avèrent quelque part remarquables [...]. En somme, le passage d'une figure du discours à une figure de style suppose le saut qualitatif du linguistique dans la sphère plus large de l'esthétique ".

Ainsi, par le contexte d'hybridation figurale qu'elle constitue, l'échelle du paradoxe peut se doter d'une rentabilité esthétique inédite dans l'univers des figures de style. Mais la « créativité » et «l'opacification du sens » ainsi renforcées peuvent amoindrir, non pas le sentiment « remarquable » de leurs « schèmes saillants » mais leur rigueur linguistique.

C'est précisément cette nécessité du contexte et ses enjeux révélateurs d'une approche plus générale des figures qu'il nous a semblé important de souligner en soulevant la question récurrente de la figuralité du paradoxe. La perspective des figures $d u$ discours nous a permis de résoudre un certain nombre de problèmes attachés au paradoxe par l'identification d'un schème matriciel, typique, mesurable mais permettant les variations et fortement polarisant pour s'opposer à une vérité apparente. Mais en retour, les spécificités du paradoxe permettent de rappeler deux problématiques fondamentales qui rendent nécessaires une analyse des figures du discours en contexte : l'ambigüité figurale, d'une part, la nuance entre rigueur de la théorie linguistique et activation d'une expressivité stylistique, d'autre part. 


\section{BIBLIOGRAPHIE}

ARISTOTE (1989) [1973] : La Rhétorique, éd. et trad. par M. Dufour, Paris, Éd. Les Belles Lettres.

BAYLE, P. (2007) [1682-1683] : Pensées diverses sur la comète, Paris, Garnier Frères.

BERgez, D., GERAUd, V. \& RobrieuX, J.-J. (1994) : Vocabulaire de l'analyse littéraire, Paris, Dunod.

Bonнomme, M. (1998) : Les Figures clés du discours, Paris, Éd. Le Seuil.

- (coord.) (2002) : « Figures du discours et ambiguïté », Semen. Revue de sémio-linguistique des textes et discours, 15.

- (2005) : Pragmatique des figures du discours, Paris, H. Champion.

- (2013) : «L'interaction entre figuralité et registres discursifs », colloque Figures du discours et contextualisation, 3-5 oct

BRUXELLES, S., DUCROT, O. \& RACCAH, P.-Y. (1995): « Argumentation and the lexical topical fields », Journal of Pragmatics, 24:1/2, p. 99-114.

CAREL, M. \& DUCROT, O. (1999a) : « Le problème du paradoxe dans une sémantique argumentative ", Langue française, 123, p. 6-26.

- (1999b) : «Les propriétés linguistiques du paradoxe : paradoxe et négation », Langue française, 123, p. 27-40.

CICERON (1971) [1930] : De L'Orateur [De oratore], éd. par H. Bornecque, trad. par E. Courbaud et H. Bornecque, Paris, Éd. Les Belles Lettres.

CLAUDEL, P. (1965) [1946] : L'CEil écoute, in : Euvres en prose, Paris, Gallimard.

CREVIER, J.-B.-L. (1765) : Rhétorique françoise par M. Crevier, Paris, Saillant et Desaint.

DEMETRIOS (2002) [1993] : Du Style [Peri Hermèneias ; trad. en latin De Elocutione], éd. et trad. par P. Chiron, Paris, Éd. Les Belles Lettres.

DOUAY-SOUBLIN, F. (2010) : « Une forme temporaire de la parole de combat : la controverse et ses "figures" antiques et médiévales ", in : L. Albert \& L. Nicolas (dirs), Polémique et rhétorique de l'antiquité à nos jours, Bruxelles/Paris, De Boeck, Duculot.

GORP, H. van et al. (2001) : Dictionnaire des termes littéraires, Paris, H. Champion.

HALM, C. (1964) [1863] : Rhetores latini minores, ex codicibus maximam partem primum adhibitis, Frankfurt-am-Main, Minerva.

LAUSBERG, H. (1998) [1960] : Handbook of literary rhetoric, A foundation for Literary Study, Leiden, Brill. mazaleyrat, J. \& MOliniÉ, G. (1989) : Vocabulaire de la stylistique, Paris, Presses universitaires de France.

MARGOLIN, J.-C. (1988) : « Le paradoxe est-il une figure de rhétorique? », Nouvelle Revue du seizième siècle, 6, p. 5-14.

MOLINIÉ, G. (2011) : Éléments de stylistique française, Paris, Presses universitaires de France.

MORIER, H. (1998) [1961] : Dictionnaire de poétique et de rhétorique, Paris, Presses universitaires de France. 
PSEUDO-ARISTOTE, (2002) [IV ${ }^{\mathrm{e}}$ siècle av. J.-C.] : Rhétorique à Alexandre, éd. et trad. par P. Chiron, Paris, Éd. Les Belles Lettres.

RICALENS POURCHOT, N. (2003) : Dictionnaire des figures de style, Paris, A. Colin.

RIFFATERRE, M. (1996) : « Paradoxe et présupposition », in : R. Landheer \& P. Smith (dirs), Le

Paradoxe en linguistique et en littérature, Genève, Droz, p. 149-171.

sushamy, H. (2013) [1981] : Les Figures de style, Paris, Presses universitaires de France.

TUTESCU, M. (1996) : «Paradoxe, univers de croyance et pertinence argumentative », in : Le

paradoxe en linguistique et en littérature, Droz, Genève, p. 79-82.

VOLTAIRE (1957) : Le Siècle de Louis XIV, chapitre XXXI, « Des Sciences », in : CEuvres historiques, Paris, Gallimard.

\section{NOTES}

1. Pour rappel: Endoxon skhếma, ámphídoxon skhếma, parádoxon skhếma, ádoxon skhếma et dusparakoloutheton skhéma (Lausberg 1998 : 31-37, § 64.3).

2. Pseudo Augustin, De Rhetorica, quae supersunt et Fortunatianus, Artis rhetorica liber III (in: Halm, 1964 : 147, 109). Leurs traductions par figurae materiarium s'opposent à celles de Genera causarum dans la Rhétorique à C. Herennius et le De Inventio de Cicéron (qui y parle aussi de qualitates) et modi causarum chez Sulpicius Victor. Victorinus soulève le problème de traduire par " genre de cause » dans son commentaire du De Inventione.

3. Il s'agit, pour F. Douay-Soublin, 2010 : 413), de «figures d'invention » contrairement aux «figures d'élocution ».

4. Julius Rufinianus, De Figuris sententiarum et elocutionis liber. De schematis lexeos. De Schematis Dianoeas; Isidore de Séville, Originum libro secundo capita quae sunt de rhetorica, chapitre «De figuris verborum et sententiarum » (in : Halm, $1964:$ 46, 505-522).

5. Néanmoins, ne pas oublier que la bipartition fondatrice permet de préciser les relevés d'occurrences en analysant des "énoncés sémantiquement paradoxaux " et des "énoncés culturellement paradoxaux » (Bruxelles, Ducrot, Raccah, 1995 : 107-108).

6. Il s'agit d'une précision de la définition comme « contre-vérité » de M. Bonhomme (2005: 56 ).

7. Il s'agit de la «forte polarisation fonctionnelle» ou renforcement du «rendement des énoncés " de la figure du discours, autrement dit, l'interaction positive entre la figure et son contexte.

8. H. Suhamy (2013: 108-117) classe le paradoxe dans son chapitre «Les figures de pensée ». Avec sa propre terminologie, G. Molinié (2011: 95) traite le paradoxe avec l'antithèse, considérant le premier comme macrostructural et la seconde comme microstructurale.

9. Rhétorique à C. Herennius (op.cit. : 148-149). Selon G. Achard, elle pourrait bien remonter aux Stoïciens. Elle a été en tout cas reprise par Cicéron, Quintilien et Rufinian jusqu'à bon nombre de manuels contemporains.

10. Exemple emprunté à M. Riffaterre (1996:152).

11. Atalide dans Bajazet, Acte III, scène 1. Exemple emprunté à M. Carel et O. Ducrot (1999a : 23).

12. Cf. M. Bonhomme (2013). Le registre est défini comme un "macro-acte illocutoire» correspondant à un certain nombre de stylèmes tonaux récurrents (" canevas scriptural ») euxmêmes s'illustrant dans des stylèmes spécifiques choisis par l'auteur (procédés et figures). Ainsi, l'ironie est aussi classée parmi les figures et non les registres.

13. Aristote (op. cit., Livre II, [1931], 1991, 1399a, 30 à 35, p. 122).

14. Ibid. (1394b, 7 à 11 \& 27 à 34, p. 107-108). 
15. Aristote (op.cit., Livre III, 1419a, 12, p. 94).

16. Récapitulation de la définition des Figures clés du discours par M. Bonhomme dans un court essai en ligne : «Figures du discours ou figure de style ? Essai de clarification » (en ligne : http:// www2.unine.ch/repository/default/content/sites/lsf2010/files/shared/abstracts/ BONHOMME.pdf, consulté le 15/05/14).

17. Fenelon, Démonstration, OC, tome I, p. 69. OC désigne désormais l'édition de Paris des CEuvres complètes de Fénelon : Euvres complètes de Fénelon, Paris, Méquignon junior et J. Leroux [puis] J. Leroux et Jouby, 1848-1852, 10 volumes. Les références aux ouvrages utiliseront les habituelles abréviations: Télémaque pour Les Aventures de Télémaque, Démonstration pour Démonstration de l'existence de Dieu, etc.

18. M. Bonhomme prend l'exemple de l'hyperbate, figure, procédé grammatical ou antifigure selon les théoriciens.

19. En effet, le premier théoricien à introduire notre figure dans un manuel français parle de "Tour de Paradoxe », mais reproduit le terme grec oxymoron en marge de son développement tandis que son exemple cicéronien développe peut-être ce qui serait plus volontiers qualifié aujourd'hui d'antithèse (Crevier, 1765 : 255-256) ! Il semble, en effet, bien délicat de séparer les phénomènes. M. Riffaterre et $C$. Fromilhague établissent que le paradoxe se distingue de l'oxymore par son incapacité à réconcilier les deux antonymes qu'il rapproche quand H. Morier, tout au contraire, le considère «moins brutal» que l'oxymore. Adopter l'une ou l'autre de ces perspectives, renvoie, quoi qu'il en soit, le jugement de figuralité à l'analyse, en contexte, du lecteur.

20. Cette figure demeure peu investie par les analyses littéraires et entendue dans des sens sensiblement différents par les théoriciens. À partir d'une «figure de pensée » de Beauzée reprise dans une «figure de mots» par Fontanier, soit le paradoxisme est un synonyme superfétatoire de paradoxe (Mazaleyrat \& Molinié, 1989 : 251), soit une figure de mots quand le paradoxe est une figure de pensée mais selon une conception étrange où il s'agit simplement d'une antithèse que l'on nomme paradoxisme alors même qu'une infinité d'autres procédés servent des paradoxes sans être renommés pour autant (Gorp et al., 2001 ; Bonhomme, 2002), soit, comme l'équivalent figural du paradoxe qui serait, lui, un concept, relançant un débat entre fond et forme, idée et réalisation formelle qui ne correspond pas à notre perspective où, justement, la figure du discours n'est pas un simple écart ou ornement mais associe une saillance à une expressivité en contexte (Morier, 1998 : 863-866, Ricalens Pourchot, 2003 ; Lausberg, 1998).

21. OC, tome VI, p. 400.

22. Lettres sur l'autorité de l'Eglise, OC, tome I, p. 211 ; Instructions sur la morale, OC, tome VI, p. 86.

23. Lettre XCII. à l'abbé de Salignac [10 décembre1710], OC, tome VII, p. 437.

24. Réponse à la Relation sur le quiétisme, OC, tome III, p. 24.

25. Lettres sur la religion, « Lettre II au duc d'Orléans » [1713], OC, tome I, p. 105.

26. Réponse à la Relation sur le quiétisme, OC, tome III, p. 17.

27. Il s'agit des Pensées sur la comète de Bayle (1683).

28. Aristote, op. cit., Livre II, 1400 a, 24 à 31, p. 125.

29. Démonstration, OC, tome I, p. 1.

30. Lettres Sur L'autorité De L'église, lettre I, OC, tome I, p. 202.

31. Dialogues des morts, XXVIII. « Denys l'ancien et Diogène ", OC, tome VI, p. 271-272. 


\section{RÉSUMÉS}

Les approches taxinomiques et hors-contexte ont instauré un doute récurrent sur la figuralité du paradoxe. Dans la perspective des figures du discours, non seulement le paradoxe trouve aisément sa place mais il devient un épiphénomène révélateur de la nécessité de penser les figures en contexte : figural et discursif. Son étude soulève, à cet égard, deux problématiques plus larges. D'une part, l'élaboration de l'échelle du paradoxe montre à quel point la théorie figurale ne peut ignorer l'hybridité constitutive de certaines figures. D'autre part, la mise au jour de l'amputation $\mathrm{du}$ contexte discursif dans la formalisation du paradoxe suggère une éventuelle dissimilation entre les plans esthétique et linguistique. Il s'agit donc moins d'évincer le paradoxe de l'univers figural que de problématiser l'univers figural à l'aune de ses spécificités.

Taxonomic and context-ignorant approaches have created a recurring doubt about considering paradox as a figure of speech. In the field of recent pragmatic theories, not only its figural nature is no longer questioned but the paradox becomes a revealing epiphenomenon of the need to think the figures in both figural and discursive contexts. Its study raises in this regard, two broader issues. On the one hand, the development of the scale of paradox shows how the figural theory cannot ignore the constitutive hybridity of some figures. On the other hand, the necessary amputation of the discursive context to speak of a paradox suggests a possible dissimilation between aesthetics and linguistics. Therefore, studying paradox does not oust it from the figural universe. On the contrary, its characteristics problematize the figural universe.

\section{INDEX}

Mots-clés : paradoxe, figure du discours, contextualisation, ambigüité

Keywords : paradox, figure of speech, contextualization, ambiguity

\section{AUTEUR}

\section{AGATHE MEZZADRI}

CEDFL (EA 3712), équipe Marge, Université Jean Moulin Lyon 3 